\title{
A Hybrid Method Based on Tissue Membrane Systems and Velocity-Position Model for Image Thresholding
}

\author{
Xinzhong Yi \\ Department of Finance and Management \\ Leshan Vocational and Technical College \\ Leshan 614000, China
}

\author{
Hong Peng \\ Center of Radio Administrator and Technology Development \\ Xihua University \\ Chengdu 610039, China \\ ph.xhu@hotmail.com
}

\author{
Zulin Zhang \\ Department of Computer Science \\ Sichuan University for Nationalities \\ Kangding 626001, China
}

\begin{abstract}
This paper proposes an image thresholding segmentation method, which combines tissue membrane systems and velocity-position model. A tissue membrane system is used as its computing framework and an improved velocity-position model is integrated as evolution rules of objects in cells. Due to parallel computing ability and inherent evolution-communication mechanism of the tissue membrane system, the presented hybrid method can effectively and efficiently find the optimal thresholds for three-level thresholding based on total fuzzy entropy. The performance of the presented hybrid method is studied with several evolutionary algorithms. Simulation results show that the presented hybrid method is superior or comparable to the other evolutionary algorithms and can be efficiently used for image thresholding.
\end{abstract}

Keywords- image thresholding; membrane computing; tissue membrane systems; total fuzzy entropy

\section{INTRODUCTION}

Image segmentation is a process of partitioning an image into units that are homogeneous with respect to one or more characteristics. Thresholding has been widely used in image segmentation. The purpose of image thresholding is to separate objects from background image or discriminate objects from objects that have distinct gray levels. In past years, a lot of thresholding methods have been reported in literature [1-3]. Bi-level thresholding segments an image into two different regions, where the pixels with gray values greater than a certain threshold are classified as object pixels while the others with gray values lesser than the threshold are regarded as background pixels. Otsu's approach [4] and Kapur's approach [5], which find the optimal thresholds by maximizing the between-class variance of gray levels or the entropy of the histogram respectively, are simple and effective in bi-level thresholding. However, the gray level histograms of many real-world images are multimodal. Therefore, multi-level thresholding has been received much attentions in recent years. Through determining multiple thresholds for an image, multi-level thresholding segments the image into several distinct regions: a background and several objects. But even though Otsu' and Kapur's methods can be extendable to multi-level thresholding in theory, they cannot efficiently be determined the optimal thresholds due to the exponential growth in computation time. In order to overcome this problem, some evolutionary algorithms have been applied to solve the multi-level thresholding problem, such as genetic algorithm (GA), particle swarm optimization (PSO) and ant colony optimization (ACO). Yin et al. [6] presented a GA-based thresholding method, where the objective function is similar to Otsu's or Kapur's functions. Cheng et al. [7] defined an method to fuzzy entropy and employed the GA to find the optimal combination of the fuzzy parameters Tao et al. [8] presented a three-level thresholding method that uses the GA to find the optimal thresholds by maximizing the fuzzy entropy. Hammouche et al. [9] proposed a multi-level thresholding method, which allows the determination of the appropriate number of threshols as well as the adequate threshold values. However, GA has some drawbacks such as slow convergence rate, premature convergence to local minima. Thus, PSO has been applied to multi-level thresholding [10-12]. In addition, Tao et al. [13] used the ACO to obtain the optimal parameters of the presented entropy-based object segmentation method.

Membrane computing [14], known as membrane systems or $\mathrm{P}$ systems, is a class of distributed parallel computing models, which is inspired by the structure and functioning of living cells as well as from the cooperation of cells in tissues, organs or populations of cells. The research results on membrane computing have made to demonstrate that most of membrane systems are powerful and efficient since they have successfully solved a lot of NP-hard problems in a linear or polynomial time [15-21]. Motivation of this paper is to develop an effective method under the framework of membrane systems to solve the 
optimal multi-level thresholding problem. Thus, a hybrid method by combining tissue membrane systems and velocity-position model that deals with a three-level image thresholding is proposed in this paper. In recent years, application of membrane systems in image processing has attracted certain attention. Díaz-Pernil et al. [22] combined the membrane structure and symport/antiport communication rules of tissue-like membrane systems to deal with homology groups of binary 2D image. Wang et al. [23] presented a bi-level image thresholding method. Zhang et al. [24] reported an object segmentation method with membrane computing.

\section{PRELIMINARIES}

\section{A. Thresholding Problem Based on Maximum Fuzzy Entropy}

Let $D=\{(i, j) \mid i=0,1, \ldots, M-1 ; j=0,1, \ldots, N-1\}, G=\{0,1, \ldots, l-$ $1\}$, where $M, N$ and $l$ are three positive integers. Suppose that $I(x, y)$ is the gray level of an image $I$ at the pixel $(x, y)$, and denote

$D_{k}=\{(x, y) \mid I(x, y)=k,(x, y) \in D\}, k=, 1, \ldots, l-1$

$h_{k}=n_{k} / M \times N$

where $n_{k}$ is the number of pixels in $D_{k}$. Thus, $0 \leq h_{k} \leq 1$, $\sum_{n=0}^{l-1} h_{k}=1$. Let $\mathrm{H}=\left\{h_{0}, h_{1}, \ldots, h_{l-1}\right\}$ be the gray histogram of the image I. $\left\{D_{0}, D_{1}, \ldots, D_{l-1}\right\}$ forms a probability partition of $D$ and its probabilistic distribution is $p_{k}=P\left(D_{k}\right)=h_{k}((k=0,1, \ldots, l-1)$.

Three-level image thresholding considered in this paper, has two thresholds, $t_{1}$ and $t_{2}$. The two thresholds segment the image $I$ into three gray levels, low gray level, middle gray level and high gray level, and the corresponding ranges are denoted by $D_{l}, D_{m}$ and $D_{h}$ respectively. Thus, $D=D_{l} \cup D_{m} \cup D_{h}, D_{l} \cap D_{m}=D_{l} \cap$ $D_{h}=D_{m} \cap D_{m h}=\varnothing$. Let $p_{l}, p_{m}$ and $p_{h}$ be the probabilistic distributions of $D_{l}, D_{m}$ and $D_{h}$, respectively, namely, $p_{l}=P\left(D_{l}\right), p_{m}=P\left(D_{m}\right), p_{h}=P\left(D_{h}\right)$. However, these probabilistic distributions are usually unknown.

For $k=0,1, \ldots, 255$, denote

$D_{k l}=\left\{(x, y) \mid I(x, y) \leq t_{1},(x, y) \in D\right\}$

$D_{k m}=\left\{t_{1} \leq(x, y) \mid I(x, y) \leq t_{2},(x, y) \in D\right\}$

$D_{k h}=\left\{(x, y) \mid I(x, y)>t_{2},(x, y) \in D\right\}$

Then, we have

$p_{k l}=P\left(D_{k l}\right)=p_{k} \times p_{l \mid k}, p_{k m}=P\left(D_{k m}\right)=p_{k} \times p_{m \mid k}$

$p_{k h}=P\left(D_{k h}\right)=p_{k} \times p_{h \mid k}$

with a constraint that $p_{l \mid k}+p_{m \mid k}+p_{h \mid k}=1 \quad(\mathrm{k}=0,1, \ldots$, 255). Thus, $p_{l}=\sum_{k=0}^{255} p_{k} \times p_{l \mid k}, p_{m}=\sum_{k=0}^{255} p_{k} \times p_{m \mid k}$ and $p_{h}=\sum_{k=0}^{255} p_{k} \times p_{h \mid k}$.

Let $\mu_{l}(k), \mu_{m}(k)$ and $\mu_{h}(k)$ denote the membership grades of a pixel belonging to $D_{l}, D_{m}$ and $D_{h}$, respectively. Then

$p_{l}=\sum_{k=0}^{255} p_{k} \times \mu_{l}(k), p_{m}=\sum_{k=0}^{255} p_{k} \times \mu_{m}(k), p_{h}=$ $\sum_{k=0}^{255} p_{k} \times \mu_{h}(k)$

The following three functions are used to approximate the membership functions above, $\mu_{l}(k), \mu_{m}(k)$ and $\mu_{h}(k)$, respectively

$\mu_{l}(k)=\left\{\begin{array}{c}1, \quad k \leq a \\ 1-\frac{(k-a)^{2}}{(c-a) \times(b-a)}, \quad a<k \leq b \\ \frac{(k-a)^{2}}{(c-a) \times(b-a)}, \quad b<k \leq c \\ 0, \quad k>c\end{array}\right.$

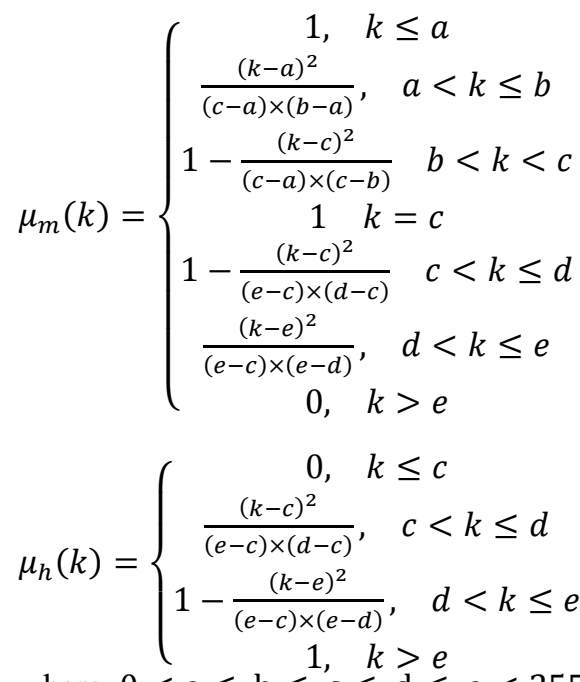

where $0<\mathrm{a} \leq \mathrm{b} \leq \mathrm{c} \leq \mathrm{d} \leq \mathrm{e}<255$.

The fuzzy entropies of above three classes are given as follows:

$H_{l}=-\sum_{k=0}^{255} \frac{p_{k} \times \mu_{l}(k)}{p_{l}} \times \ln \left(\frac{p_{k} \times \mu_{l}(k)}{p_{l}}\right)$
$H_{m}=-\sum_{k=0}^{255} \frac{p_{k} \times \mu_{m}(k)}{p_{m}} \times \ln \left(\frac{p_{k} \times \mu_{m}(k)}{p_{m}}\right)$
$H_{h}=-\sum_{k=0}^{255} \frac{p_{k} \times \mu_{h}(k)}{p_{h}} \times \ln \left(\frac{p_{k} \times \mu_{h}(k)}{p_{h}}\right)$

Thus, the total fuzzy entropy is computed as folows

$H(a, b, c, d, e)=\mathrm{H}_{l}+\mathrm{H}_{m}+\mathrm{H}_{h}$

Based on maximum fuzzy entropy principle, the threelevel image thresholding problem can be formulated as an optimization (maximization) problem where the total fuzzy entropy is used for the objective function

$\max _{w} J(w)=\max _{w}\left[H(a, b, c, d, e)=\mathrm{H}_{l}+\mathrm{H}_{m}+\mathrm{H}_{h}\right]$

where $w=(a, b, c, d, e)$ denotes the parameters to be optimized. In this paper, membrane systems will be used to optimize these parameters.

Note that threshold $t_{1}$ is the intersection point of curves $\mu_{l}(k)$ and $\mu_{m}(k)$, while threshold $t_{2}$ is the intersection point of curves $\mu_{m}(k)$ and $\mu_{h}(k)$. Therefore, according to Eqs.6)-(8), the two thresholds can be calculate as follows:

$t_{1}=\left\{\begin{array}{l}a+\sqrt{(c-a) \times(b-a) / 2}, \quad(a+c) / 2 \leq b \leq c \\ c-\sqrt{(c-a) \times(c-b) / 2}, a \leq b \leq(a+c) / 2\end{array}\right.$

$t_{2}= \begin{cases}c+\sqrt{(e-c) \times(d-c) / 2}, & (c+e) / 2 \leq d \leq e \\ e-\sqrt{(e-c) \times(e-d) / 2}, & c \leq d \leq(c+e) / 2\end{cases}$

\section{B. Abbreviations and Acronyms}

Tissue membrane systems are a kind of membrane systems, which are inspired from the behaviour of several single-membrane cells evolved in a common environment. A tissue membrane system can be viewed as a net of processors, which deal with the objects and communicates them according to the channels assigned in advance [25]. We briefly review the definition and inherent mechanism of tissue membrane systems as follows.

A tissue membrane system of degree $\$ \mathrm{~m} \$$ is a construct

where 
(1) $w_{i}$ is finite set of objects initially present in cell $i, 1 \leq i \leq$ $m$

(2) $R_{i}$ is finite set of evolution rules of the form $u \rightarrow v$ in cell $i, 1 \leq i \leq m$;

(3) $\mathrm{R}^{\prime}$ is finite set of communication rules of the forms $(i, u / v, j)$ and $(i, u / \lambda, 0), i, j=1,2, \ldots, m, i \neq j$

(4) $i_{o}$ indicates the output region of the system.

The tissue membrane system consists of $m$ cells and the region outside the $q$ cells is called the environment. Each cell contains several objects. $w_{1}, \ldots, w_{m}$ describe multisets of objects of the $d$ cells, respectively. Evolution rule of the form $u \rightarrow v$ indicates that object $u$ will be evolved to object $v$. Communication rule of the form $(i, u / v, j)$ means that object $u$ in cell $i$ and object $v$ in cell $j$ are interchanged. In particular, the communication rule of the form $(i, u / \lambda, 0)$ indicates that object $u$ in cell $i$ is transported into the environment.

As usual in the framework of membrane computing, every cell as a computing unit works in parallel.

This system is working as follows: it starts with initial multisets of objects $w_{1}, \ldots, w_{m}$; in each computing step, objects in each cell are evolved by evolution rules, and then its some objects are transported to all the connected cells and/or are sent to the environment by communication rules; the evolution-communication procedure is repeated constantly until halting condition is reached, thus the system halts. When the system halts, final computing results are in the output region of the system.

\section{PROPOSED THRESHOLDING METHOD}

The proposed thresholding method is designed by combining a tissue membrane system of $m$ cells and velocity-position model. Fig .1 shows the tissue membrane system of $m$ cells. The role of cells labeled by $1,2, \ldots, m-1$ is to evolve the objects in the system, thus, the cells are called the evolution cells. The cell labelled by $\$ \mathrm{~m} \$$ is used to store the best object found in the whole system so far, called the store cell. Assume that each evolution cell contains $n$ objects, which will be evolved by evolution rules. In this work, velocity-position model is used as evolution rules in evolution cells. Each evolution cell can memory its current best object (with highest fitness value), which is called the local best object, denoted $X_{\text {lbest }}$. The store cell has no evolution rules and contains only an object, which is called the global best object, denoted $X_{\text {gbest }}$. The global best object will be updated constantly by the objects that are communicated from evolution cells. The object communication is achieved by communication rules between cells. In addition, the store cell is also output region of the system.

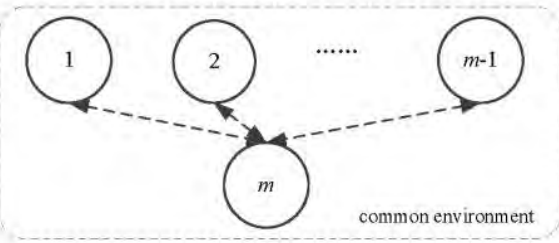

Figure 1. Membrane structure of the used tissue membrane system.

In this work, each object is designed as a fivedimensional vector, $\mathrm{X}=\left(x_{1}, x_{2}, x_{3}, x_{4}, x_{5}\right)$, where $x_{1}, x_{2}$, $x_{3}, x_{4}$ and $x_{5}$ correspond to five segmentation parameters, $a, b, c, d$, and $e$, respectively. Therefore, each object in fact expresses a group of feasible segmentation thresholds in search space. The total fuzzy entropy (i.e., Eq. (10)) will be regarded as fitness function of object in the system to evaluate the quality of each object, i.e., Fitness = $H(a, b, c, d, e)$.

In order to clear understand the proposed thresholding method, its computing procedure is described as follows.

\section{Step 1. Initialization}

For each evolution cell, $n$ initial objects are generated.

Each initial object is composed of an integer vector with 5 components randomly produced in $[0,255]$. Note that if a randomly generate object do not hold the increasing order, $0<\mathrm{a} \leq \mathrm{b} \leq \mathrm{c} \leq \mathrm{d} \leq \mathrm{e}<255$, we can re-compute its components as follows:

$$
\begin{aligned}
& \mathrm{c}^{\prime}=\mathrm{c}, \mathrm{b}^{\prime}=\mathrm{c}^{\prime} \times\left(\frac{\mathrm{b}}{255}\right), \mathrm{a}^{\prime}=\mathrm{b}^{\prime} \times\left(\frac{\mathrm{a}}{255}\right) \\
& \mathrm{d}^{\prime}=\mathrm{c}^{\prime}+\left(255-\mathrm{c}^{\prime}\right) \times\left(\frac{\mathrm{d}}{255}\right) \\
& \mathrm{e}^{\prime}=\mathrm{d}^{\prime}+\left(255-\mathrm{d}^{\prime}\right) \times\left(\frac{\mathrm{e}}{255}\right)
\end{aligned}
$$

\section{Step 2. Evolution rules}

The used tissue membrane system searches the optimal segmentation parameters for optimal image thresholding problem (11) by the evolution of objects in cells. Several evolution cells are designed to co-evolve objects in the system, thus this will accelerate the exploitation of optimal segmentation parameters. In this work, the known velocity-position model in particle swarm optimization [26, 27] is introduced as evolution rules of objects, however, an improved velocity-position model is developed according to special structure and communication mechanism of the tissue membrane system.

Assuming that $X_{i}$ is $i$ th object in a cell, the improved velocity-position model can be described as follows:

$$
\begin{aligned}
& V_{i}=w X_{i}+c_{1} r_{1}\left(P_{i}-X_{i}\right)+c_{2} r_{2}\left(\mathrm{X}_{\text {lbest }}^{i}-X_{i}\right)+ \\
& c_{3} r_{3}\left(\mathrm{X}_{\text {gbest }}^{i}-X_{i}\right) \\
& X_{i}=\text { floor }\left(X_{i}+V_{i}\right)
\end{aligned}
$$

where $P_{i}$ is the best position of object $X_{i}$ found so far, $w$ is inertia weight, $c_{1}, c_{2}, c_{3}$ are learning factors, $r_{1}, r_{2}, r_{3}$ are three random real numbers in $[0,1]$, and $f \operatorname{loor}(\cdot)$ is a rounded function. In the implementation, we use the following decreasing strategy of inertia weight:

$$
w=w_{\max }-\left(w_{\max }-w_{\min }\right) t / t_{\max }
$$

where $w_{\max }$ and $w_{\min }$ are maximum value and minimum value of inertia weight respectively, and $t_{\max }$ is maximum computing step number (or maximum iteration number).

\section{Step 3. Communication rules}

The communication of objects is an inherent mechanism of the tissue membrane system, which is used to exchange and share objects between evolution cells and the store cell, shown in Fig .1. The communication mechanism is achieved by the following communication rule:

$$
\left(i, X_{\text {lbest }}^{i} / \mathrm{X}_{\text {gbest }}^{i}, m\right), i=1,2, \ldots, m-1
$$

This rule means that the local best object $X_{\text {lbest }}^{i}$ in cell $i$ and the global best object $X_{\text {gbest }}^{i}$ in the store cell are exchanged. In other words, each evolution cell will receive the global best object from the store cell, and then transports its local best object into the store cell and updates the global best object. 


\section{Step 4. Halting and output}

The maximum execution step number is employed as halt condition in the proposed thresholding method based on tissue membrane systems. When the system halts, the global best object $X_{\text {gbest }}^{i}$ in output region is regarded as the output of entire system, namely, the optimal segmentation parameters.

\section{EXPERIMENTAL RESULTS}

The applicability and efficiency of the proposed thresholding method in image segmentation has be evaluated on six standard test images, which are Hunter, Lena, Peppers, Butterfly, Baboon and House respectively, shown in Fig.2(a)-(f). The test images are with size $512 \times 512$.

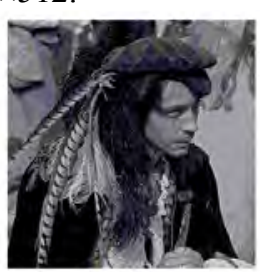

(a)

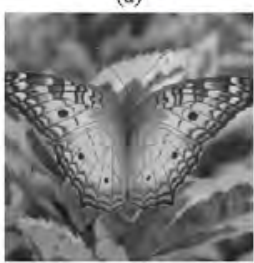

(d)

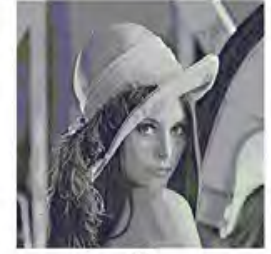

(b)

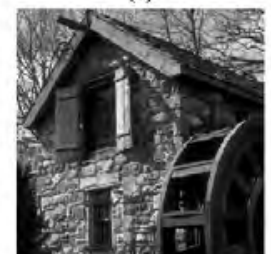

(e)

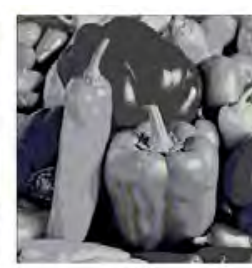

(c)

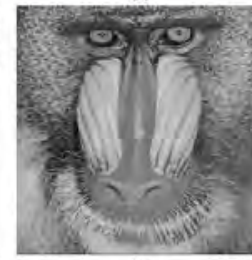

(f)
Figure 2. Three test images: (a) Hunter; (b) Lena; (c) Peppers; (d) Butterfly; (e) Baboon; (f) House.

In experiments, input parameters of the proposed thresholding method are given as follows:

(i) The used tissue membrane system includes three evolution cells and a store cell $(m=4)$, where the number of objects contained in each evolution cell is $n=50$, and the maximum execution step number $i$, $t_{\max }=100$;

(ii) In the used velocity-position model, $c_{1}=c_{2}=c_{3}=$ 1.0 , and $w$ linearly varies from 0.9 to 0.4 .

In order to illustrate segmentation performance of the proposed three-level thresholding method, its segmentation results are compared with the results obtained by PSObased and GA-based methods as well as Lou's method [28], respectively.

For the PSO-based method, standard position-velocity model is employed and its input parameters are given: population size $N P=30$, maximum generation number $t_{\max }=100, c_{1}=c_{2}=1.0$, and $w$ linearly varies from 0.9 to 0.4 . For the GA-based method, its input parameters are given: population size $N P=30$, crossover probability $p_{c}=0.6$ mutation rate $p_{m}=0.01$ and maximum generation number $t_{\max }=100$.

Fig .2 only shows three-level segmentation results on three of six the test images (due to space limit) for the proposed thresholding method based on tissue membrane systems (in short, membrane systems), PSO-based method GA-based method and Luo's method, respectively. The optimal segmentation thresholds are provided in Table 1. From the Fig .2, we can see that results of the proposed method are better than that of PSO-based method and significantly outperform that of GA-based method and Lou's method. The results demonstrate the applicability of the proposed thesholding method based on tissue membrane systems.

TABLE I. The Optimal SEgmentation ThRESHOLDS OBTAINED BY DIFFERENT METHODS

\begin{tabular}{|l|l|l|l|l|}
\hline Images & $\begin{array}{l}\text { Membrane } \\
\text { system }\end{array}$ & PSO & GA & Luo [28] \\
\hline Lunter & 86,179 & 82,183 & 73,179 & 52,194 \\
\hline Lena & 98,165 & 99,166 & 103,168 & 76,189 \\
\hline Peppers & 76,152 & 80,145 & 85,151 & 69,177 \\
\hline Butterfly & 96,153 & 100,149 & 111,179 & 90,185 \\
\hline Baboon & 95,150 & 94,154 & 86,165 & 78,174 \\
\hline House & 56,129 & 59,130 & 49,141 & 40,156 \\
\hline
\end{tabular}

In order to investigate the efficiency, all methods are compared based on the average CPU time (in seconds) taken to converge the solution. Comparison results of all methods provided in Table 2. Form Table 2, it is clear that the proposed thresholding method based on tissue membrane systems has fast convergence compared with PSO-based and GA-based methods and Lou's method. The results illustrate that the proposed thresholding method based on tissue membrane systems is more efficient and effective than PSO-based and GA-based methods as well as Lou's method for three-level image thresholding.

TABLE II. COMPARISON OF CPU TIME (IN SECONDS) FOR DIFFERENT

\begin{tabular}{|l|l|l|l|l|}
\hline Images & $\begin{array}{l}\text { Membrane } \\
\text { system }\end{array}$ & PSO & GA & Luo [28] \\
\hline Lunter & 7.862 & 9.521 & 11.973 & 83.552 \\
\hline Lena & 7.558 & 9.136 & 11.654 & 82.294 \\
\hline Peppers & 6.975 & 9.849 & 12.117 & 80.195 \\
\hline Butterfly & 7.186 & 9.772 & 11.995 & 84.017 \\
\hline Baboon & 7.193 & 9.685 & 11.672 & 81.375 \\
\hline House & 7.614 & 9.431 & 11.846 & 83.782 \\
\hline
\end{tabular}

\section{CONCLUSIONS}

In this paper, we have presented a fast three-level thresholding methos based on tissue membrane systems, which employs the total fuzzy entropy as the evaluation criterion. In order to effectively exploit the optimal segmentation thresholds, a tissue membrane system of \$m\$ cells was designed, which allows multiple cells to coevolve the objects of the system, and an improved velocity-position model was developed as evolution rules of these cells. The evolution-communication mechanism not only effectively accelerates the speed of convergence but also enhances the diversity of objects in the system. The proposed image thresholding method based on tissue membrane systems has been tested on several standard images and compared with GA-based and PSO-based methods as well as a traditional method. The experimental results showed the proposed image thresholding method outperforms the other methods in terms of the applicability and computation efficiency. Further work is application of membrane systems in various types of image processing problem.

\section{ACKNOWLEDGMENT}

This work was partially supported by the Sichuan Key Technology Research and Development Program (No. 2013GZX0155), China. 

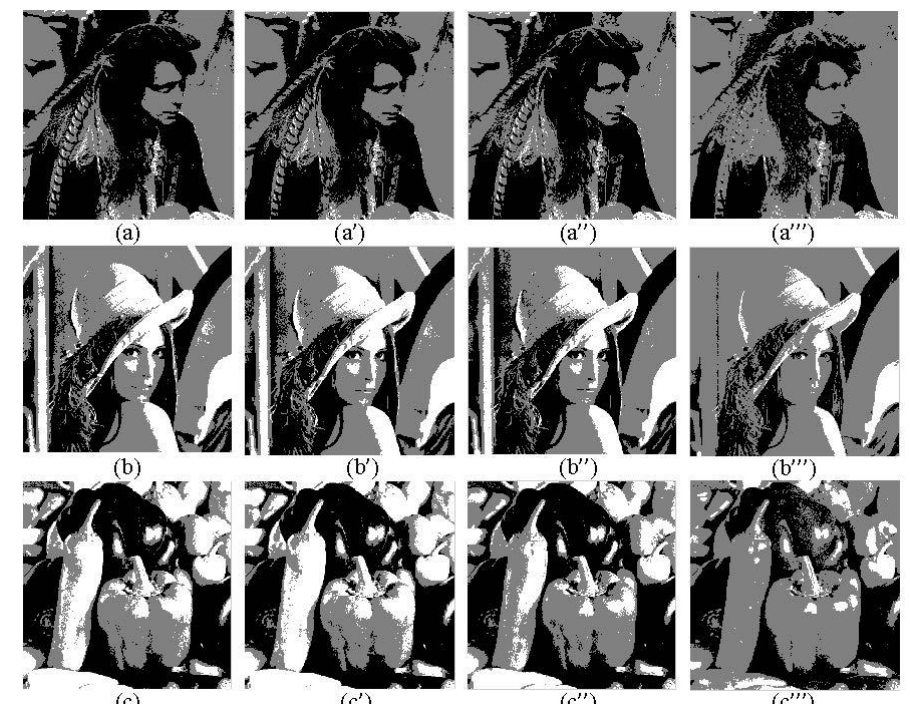

Figure 3. Three-level thresholding images obtained by different methods: (a)-(c) tissue membrane systems; (a')-(c') PSO; (a")-(c") GA; (a"')-(c'") Luo et al [28].

\section{REFERENCES}

[1] P. K. Sahoo, S. Soltani, A. K. C. Wong, Y. C. Chen, "A survey of thresholding techniques," Comput. Vis. Graph. Image Process., vol. 41, no 2, 1988, pp. 233-260.

[2] A. Pikaz, A. Averbuch, "Digital image thresholding based on topological stable state", Pattern Recognit., vol. 29, no. 5, 1996, pp. 829-843.

[3] L. K. Huang, M. J. Wang, "Image thresholding by minimizing the measure of fuzziness," Pattern Recognition, vol. 28, 1995, pp. 4151.

[4] N. Otsu, "A threshold selection method from gray level histograms," IEEE Transactions on Systems, Man and Cybernetics SMC, vol. 9, no. 1, 1979, pp. 62-66.

[5] J. N. Kapur, P. K. Sahoo, A. K. C. Wong, "A new method for graylevel picture thresholding using the entropy of the histogram," Computer Vision, Graphics and Image Processing, vol. 29, no. 3, 1985, pp. 273-285.

[6] P. Y. Yin, "A fast scheme for optimal thresholding using genetic algorithms," Signal Processing, vol. 72, 1999, pp. 85-95.

[7] H. D. Cheng, Y. H. Chen, Y. Sun, "A novel fuzzy entropy approachto image enhancement and thresholding," Signal Processing, vol. 75, 1999, pp. 277-301.

[8] W. B. Tao, J. W. Tian, J. Liu, "Image segmentation by three-level thresholding based on maximum fuzzy entropy and genetic algorithm," Pattern Recognit. Lett., vol. 24, 2003, pp. 3069-3078.

[9] K. Hammouche, M. Diaf, P. Siarry, "A multilevel automatic thresholding method based on a genetic algorithm for a fast image segmentation," Computer Vision and Image Understanding, vol. 109, 2008, pp. 163-175.

[10] E. Zahara, S. K. S. Fan, D. M. Tsai, "Optimal multi-thresholding using a hybrid optimization approach," Pattern Recognit. Lett., vol. 26, 2005, pp. 1085-1095.

[11] M. Maitra, A. Chatterjee, "A hybrid cooperative-comprehensive learning based PSO algorithm for image segmentation using multilevel thresholding," Expert Systems with Applications, vol. 34, 2008, pp. 1341-1350.

[12] H. Gao, W. B. Xu, J. Sun, Y. L. Tang, "Multilevel thresholding for image segmentation through an improved quantum-behaved particle swarm algorithm," IEEE Trans. On Instrumentation and Measurement, vol. 59, no. 4, 2010, pp. 934-946.

[13] W.B. Tao, H. Jin, L. M. Liu, "Object segmentation using ant colony optimization algorithm and fuzzy entropy, Pattern Recognit," Lett., vol. 28, no. 7, 2008, pp. 788-796.

[14] Gh. Păun, "Computing with membranes," Journal of Computer System Sciences, vol. 61, no. 1, 2000, pp. 108-143.
[15] Gh. Păun, G. Rozenberg, A. Salomaa, "The Oxford Handbook of Membrance Computing," Oxford Unversity Press, New York, 2010.

[16] Gh. Păun, M.J. Pérez-Jiménez, "Membrane computing: brief introduction, recent results and applications," BioSystem, vol. 85, 2006, pp. 11-22.

[17] J. Wang, L. Zhou, H. Peng, G. X. Zhang, "An extended spiking neural P system for fuzzy knowledge representation," International Journal of Innovative Computing, Information and Control, vol. 7, no. 7A, 2011, pp. 3709-3724.

[18] H. Peng, J. Wang, M.J. Pérez-Jiménez, H. Wang, J. Shao, T. Wang, "Fuzzy reasoning spiking neural P system for fault diagnosis," Information Sciences, vol. 235, 2013, pp. 106-116.

[19] J. Wang, P. Shi, H. Peng, M.J. Pérez-Jiménez, T. Wang, "Weighted fuzzy spiking neural P systems," IEEE Transactions on Fuzzy Systems, vol. 21, no. 2, 2013, pp. 209-220

[20] T.Y. Nishida, "An application of P-system: a new algorithm for NP-complete optimization problems," In: Proc. 8th World MultiConference on Systemics, Cybernetics and Informatics, 2004, pp. 109-112.

[21] L. Huang, I. H. Suh, A. Abraham, "Dynamic multi-objective optimization based on membrane computing for control of timevarying unstable plants," Information Sciences, vol. 181, 2011, pp. 2370-2391.

[22] D. Díaz-Pernil, M.A. Gutiérrez-Naranjo, P. Real, V. SánchezCanales, "A cellular way to obtain homology groups in binary 2D images," Eighth Brainstorming Week on Membrane Computing, 2010, pp. 89-100

[23] H. Wang, H. Peng, J. Shao, "A thresholding method based on P systems for image segmentation," ICIC Express Letters, vol. 6, no 1, 2012, pp. 221-227.

[24] Z. Zhang, H. Peng, "Object segmentation with membrane computing," Journal of Information $\backslash \&$ Computational Science, vol. 9, no. 17, 2012, pp. 5417-5424.

[25] R. Freund, Gh. Păun, M.J. Pérez-Jiménez, "Tissue-like P Systems with Channel-states," Theoretical Computer Science, vol. 330, 2005, pp. 101--116.

[26] J. Kennedy, R.C. Eberhart, "Particle swarm optimization," In: Proc. IEEE Int. Conf. Neural Networks, Perth., Perth, Australia 4, 1995, pp. 1942-1948.

[27] R. C. Eberhart, J. Kennedy, Y. H. Shi, Swarm Intelligence, San Mateo, Morgan Kaufmann, CA, 2001

[28] X. Luo, J. Tian, "ICM method for multi-level thresholding using maximum entropy criterion," In: Proc. of Internat. Conf. on Image Analysis and Processing, 1999, pp. 108-113 